\title{
Real-Time 3D Transesophageal Echocardiographic Guidance of Prosthetic Valve Paravalvular Leak
}

\author{
Joseph M. Venturini MD, Anuj Mediratta MD, Karima Addetia MD, Sandeep Nathan MD, Atman P. Shah MD, \\ Roberto M. Lang MD* \\ University of Chicago Medical Center, Section of Cardiology, Chicago, Illinois, USA \\ The first two authors contributed equally to the manuscript.
}

NOTE: This manuscript includes videos. Not all PDF readers support video. For desktop computers we recommend using Adobe Acrobat Reader. To view videos within a PDF on an iPad we recommend viewing in ezPDF Reader or PDF Expert. On desktop computers you may view videos full screen by clicking on a video, right click on the video, then choose Full Screen Multimedia.

\begin{abstract}
Paravalvular leak (PVL), defined as retrograde blood flow adjacent to an annuloplasty ring (Figure $1 \mathrm{a}$, Video 1 ) or prosthetic valve (Figure $1 \mathrm{~b}$, Video 2 ), is a rare but serious complication of heart valve surgery. Though most PVLs are asymptomatic, $1-5 \%$ of patients develop serious clinical consequences such as heart failure, endocarditis, or hemolysis $[1,2]$. Surgical repair may be necessary in severe cases, however for those who are at high surgical risk, a percutaneous approach can be performed to occlude these defects [1,3-9]. Real-time three-dimensional
\end{abstract}

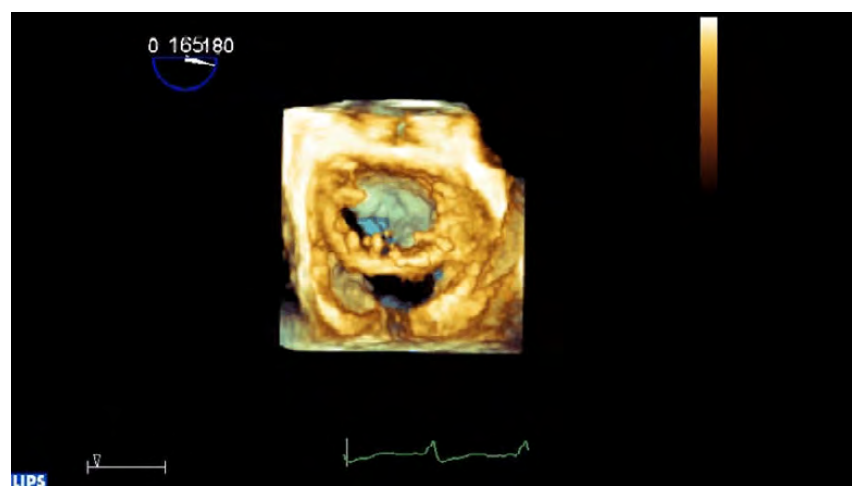

Video 1. Annuloplasty ring dehiscence.

(c) 2015 Journal of Structural Heart Disease Published by Science International Corp. ISSN 2326-4004

Fax +1 2037853346

E-Mail: jshd@scienceinternational.org

http://structuralheartdisease.org/
Accessible online at:

http://structuralheartdisease.org/ transesophogeal echocardiography (3DTEE) during percutaneous closure procedures is invaluable for intra-procedural guidance. In this article, we will review the literature and outline two cases where real-time 3DTEE guidance was critical for successful closure of symptomatic PVL.

Copyright (c) 2015 Science International Corp.

\section{Key Word}

3D TEE Guidance

\section{Cases}

Case 1

A 64-year-old female with history of congenital heart disease requiring ostium primum atrial septal defect (ASD) repair and multiple mitral valve replacements presented with worsening heart failure. Most recently, she underwent a third mechanical mitral valve replacement (in the setting of severe $P V L)$, mechanical aortic valve replacement, tricuspid valve repair, and ventricular septal defect repair. Since her last surgery, she developed New York Heart

* Corresponding Author:

Roberto M. Lang, MD, FASE Section of Cardiology

University of Chicago Medical Center

5841 South Maryland Avenue, M512, Chicago, Illinois 60637, USA

Tel.: +1 773 702-1842, Fax: +1 773 702-1034, E-Mail: rlang@medicine.bsd.uchicago.edu 

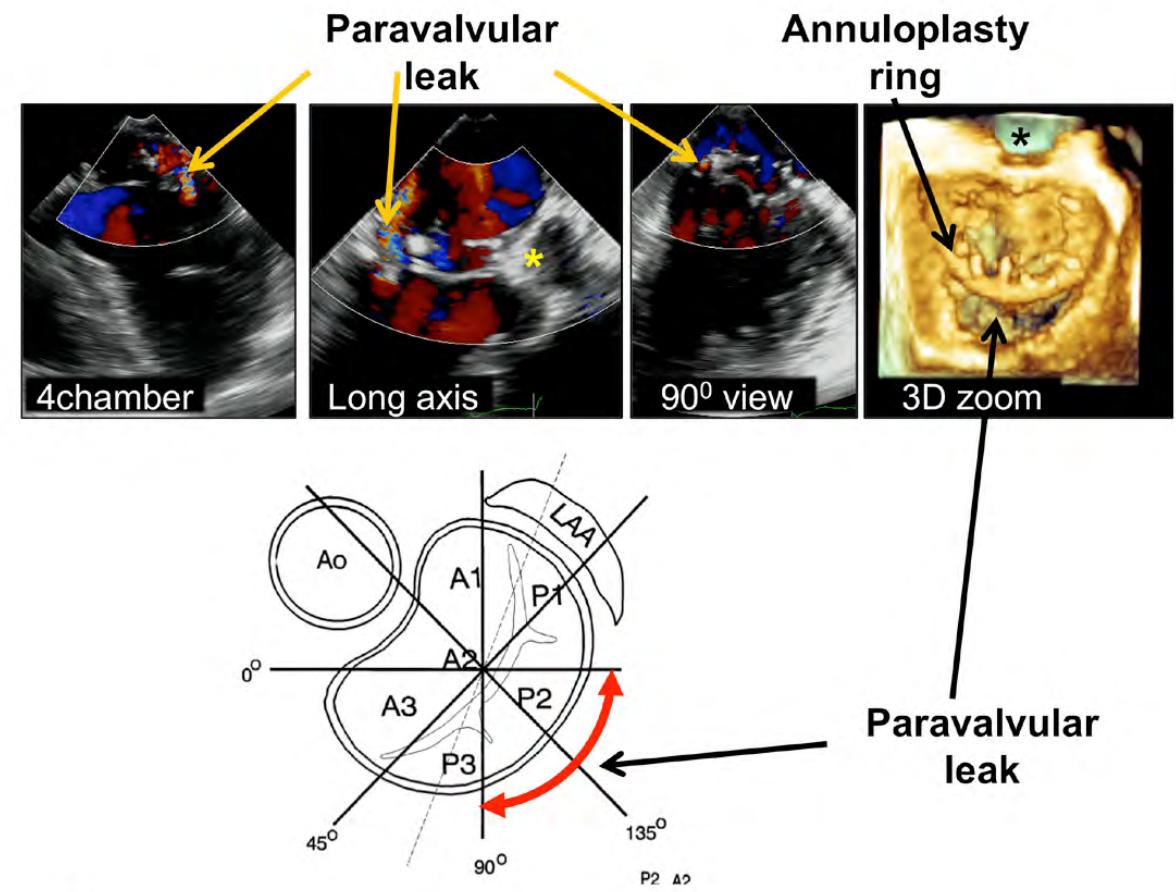

Figure 1a. Paravalvular leak adjacent to an annuloplasty ring. 2D TEE 4-chamber (top row, far right), long axis (top row, center right), and 90-degree views (top row, center left) depict the case of a patient with extensive paravalvular leak spanning across three viewing planes along the posterior portion of the mitral annulus (bottom figure). This is well seen on 3D image (top row far right).

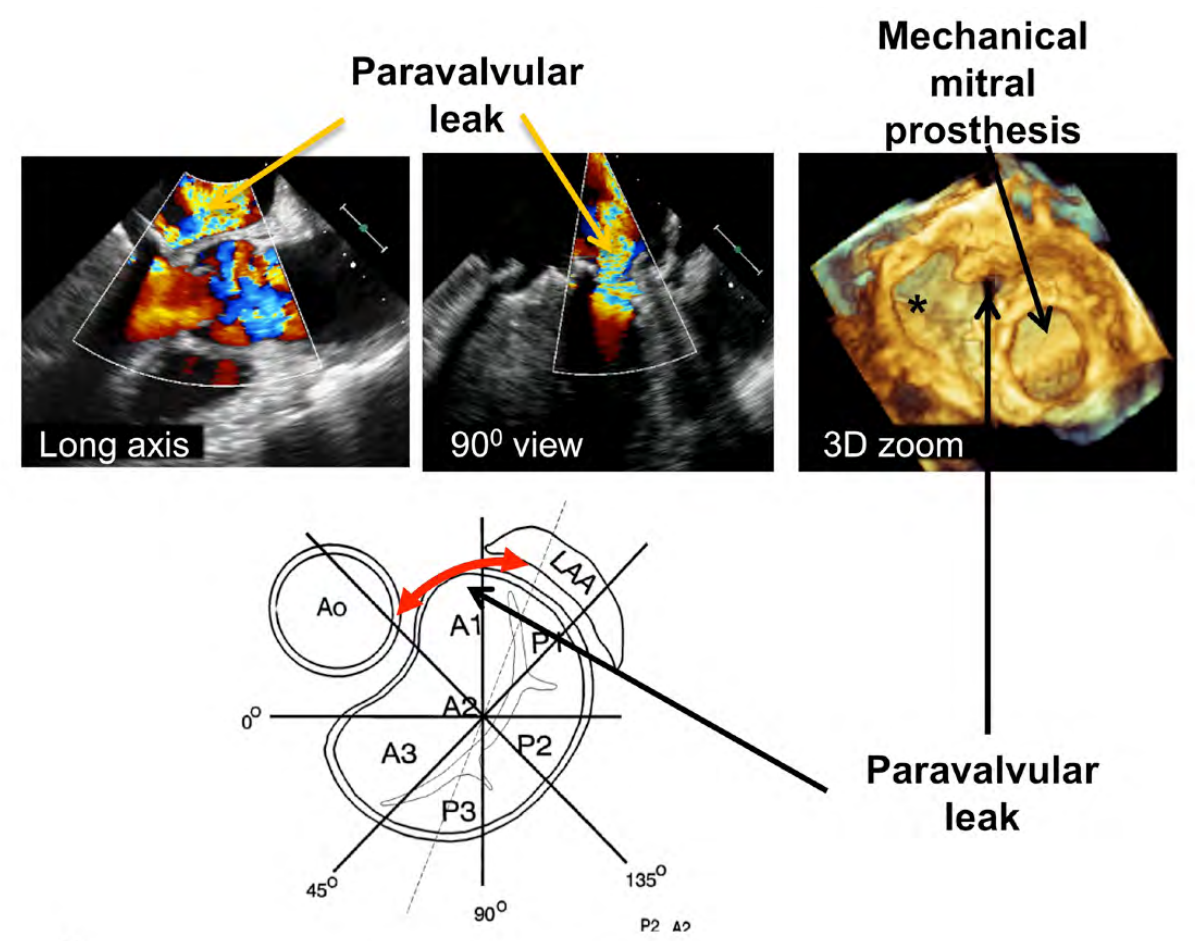

Figure 1b. Paravalvular leak adjacent to a prosthetic valve. 2D TEE long axis (top row, far right), and 90 degree views (top row, center) depict the case of a patient with paravalvular leak spanning across two viewing planes along the anterior wall of the mitral annulus on the side of the aorta and the left atrial appendage (bottom figure showing approximate location of the leak). This is well seen on 3D image (top row far right). Asterisk shows the location of the left atrial appendage. 


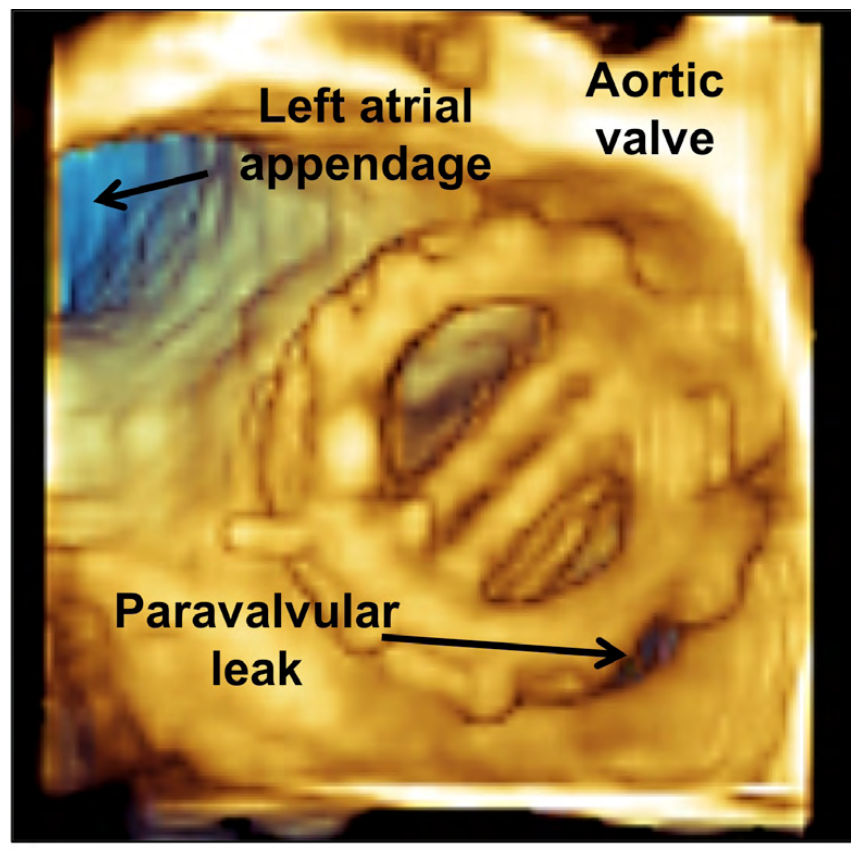

Figure 2. $3 \mathrm{D}$ zoom view of the mechanical mitral prosthesis with paravalvular leak as noted. Aortic valve is in the 12 o'clock position.

Association (NYHA) Class III heart failure symptoms and atrial fibrillation. Diagnostic evaluation including right heart catheterization and TEE revealed severe pulmonary hypertension (PA pressure $84 / 34 \mathrm{mmHg}$ with a mean of $50 \mathrm{~mm} \mathrm{Hg}$ ) and an area of partial dehiscence of the prosthetic mitral valve with a large PVL. Due to her worsening heart failure and history of multiple sternotomies, surgical repair was deemed to be associated with prohibitive risk and a percutaneous approach was planned.

Due to location of the defect (Figure 2, Video 3) and the presence of a mechanical aortic valve, a trans-apical approach was chosen for percutaneous closure over a trans-septal or retrograde aortic approach. After apical access was obtained, the paravalvular defect was identified and crossed with a 0.035inch $x 150 \mathrm{~cm}$ Terumo straight stiff glide wire (Terumo Medical, Somerset, New Jersey, USA) (Figure 3) with 3DTEE guidance. Initially, an 8-mm muscular ventricular septal defect occluder (St. Jude Medical, St. Paul, Minnesota, USA) was positioned and deployed into the large defect. On 3DTEE and fluoroscopy, there was evidence of entrapment of a mechanical mitral valve leaflet and the device was retrieved. Next, a

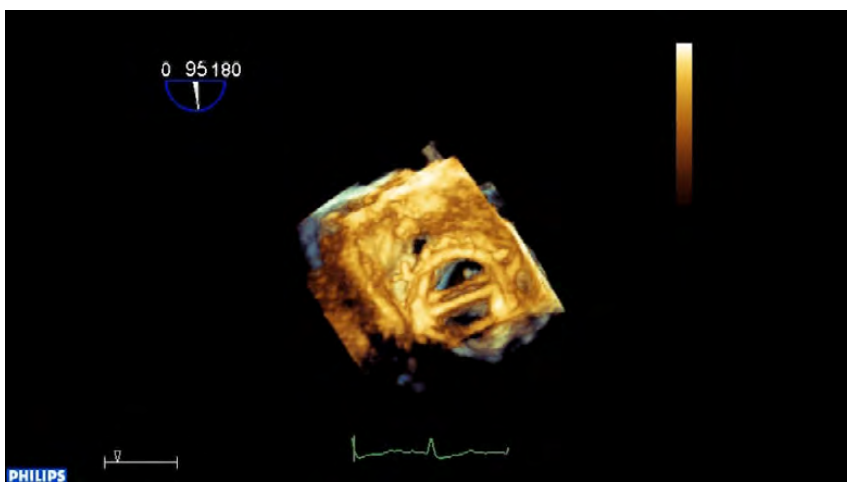

Video 2. Mechanical mitral prosthetic dehiscence.

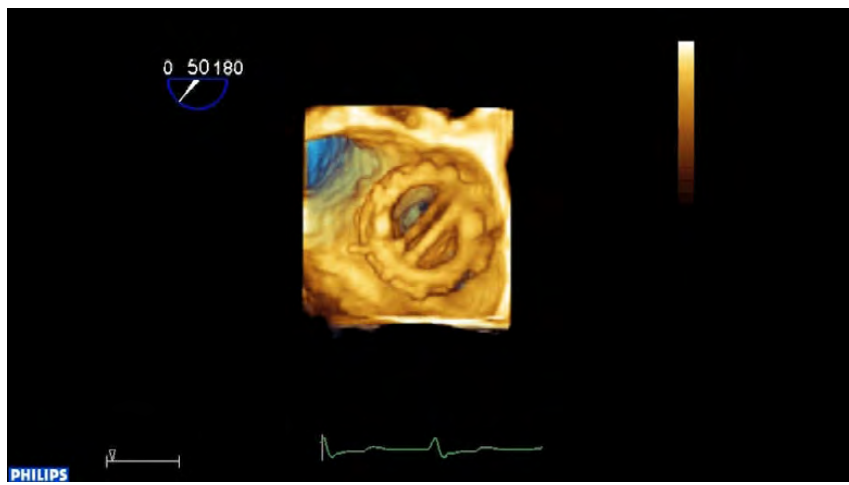

Video 3. Case 1: Mechanical mitral prosthesis with paravalvular leak in the 4 o'clock position.

$6 \mathrm{~mm}$ muscular VSD occluder device (St. Jude Medical, St. Paul, Minnesota, USA) was placed into the defect without compromise of leaflet mobility on TEE (Figure 4, Video 4) and fluoroscopy. TEE confirmed closure of the large PVL. Using transthoracic echocardiographic guidance, the trans-apical puncture site was closed with a $6 \mathrm{~mm} / 4 \mathrm{~mm}$ Amplatzer Duct Occluder (St. Jude Medical, St. Paul, Minnesota, USA). The patient tolerated the procedure well and was discharged home 3 days later.

\section{Case 2}

A 72-year-old male with history of rheumatic heart disease requiring mechanical mitral and aortic valve replacements was transferred for consideration of repeat mitral valve replacement. He was initially hospitalized with acute pulmonary edema and severe hemolytic anemia requiring blood transfusions. Upon transfer there was significant volume overload, and severe intravascular hemolysis as evidenced by low 

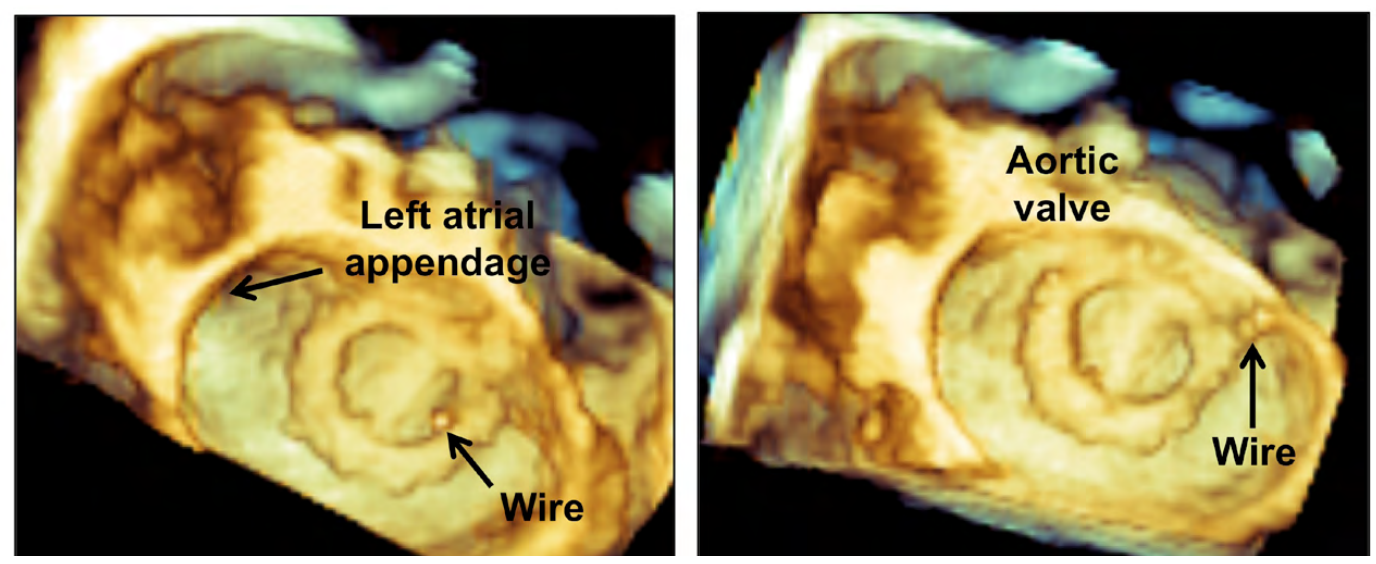

Figure 3. 3D full volume rendering of the mechanical mitral prosthesis with the guide wire in the wrong location (left panel) and guide wire in the right position (right panel). Aortic valve is in the 12 o'clock position.

hemoglobin and haptoglobin, elevated lactate dehydrogenase, and an unconjugated hyperbilirubinemia. The etiology of the patient's heart failure and hemolytic anemia were determined to be secondary to severe PVL of the mechanical mitral valve on TEE. Due to severe deconditioning and comorbidities patient was deemed high surgical risk, and a percutaneous approach was preferred.

Given the anatomy and location of the PVL, a transapical approach was chosen to facilitate crossing and closure of the defect. With 3DTEE guidance, the PVL anatomy was identified showing two distinct PVLs with the larger dehiscence located along the inferolateral aspect of the valve annulus (Figure 5). With TEE guidance, the defect was crossed with a guidewire and a $6 \mathrm{~mm}$ Amplatzer VSD occluder device was placed across the defect, however, TEE and fluoroscopic imaging showed evidence of entrapment of the posterior mechanical mitral valve leaflet (Video 5, Video 6, Video 7). The occluder device was removed and a $4 \mathrm{~mm}$ Amplatzer VSD occluder device was positioned across the defect and deployed with resolution of PVL demonstrated by TEE. The trans-apical puncture site was closed with a $6 \mathrm{~mm} / 4 \mathrm{~mm}$ Amplatzer Duct Occluder.

\section{Discussion}

Incidence

The incidence of PVL post heart valve surgery is 5 to $17 \%$ [10-12]. PVL occur more commonly in patients with prosthetic mitral compared to those with prosthetic aortic valves. The estimated incidence ranges between $7-32 \%$ in the mitral position and $2-10 \%$ in the aortic position, with the most clinically significant complications occurring in the mitral position $[10,11,13]$. The majority of PVLs are frequently single, but may be multiple in $27 \%$ of patients [14]. It is unclear whether PVLs occur more frequently in bioprosthetic valves vs. mechanical valves [10].

\section{Etiology and Natural History}

PVL occur as a consequence of an incomplete seal between the ring of the implanted valve and the surrounding cardiac tissue. Known risk factors for PVL occurrence include annular calcification, small prosthetic size, inadequate suturing technique, and infection [15]. PVLs that are identified soon after implantations are most often secondary to technical complications of the operation; in contrast, PVLs identified late after surgery are most frequently a consequence of infectious endocarditis or secondary to significant annular calcification [16].

PVL size correlates directly with onset of symptoms; with larger size leaks resulting in heart failure symptoms. Smaller PVL may create high velocity jets into the low pressure left atrium. These jets may collide with structures such as the limbus, which separates, the appendage from the left superior pulmonic vein, resulting in hemolysis. The number of PVLs does not appear to correlate with symptoms, but increasing numbers of leaks increase the risk of associated 
hemolysis [14]. With follow-up, leaks may increase or decrease in size, or, less commonly, may spontaneously close $[13,17,18]$. Importantly, the presence of PVL results in turbulent blood flow thereby augmenting the risk for the development of infective endocarditis in the presence of bacteremia. If regurgitant flow is significant and not corrected, the natural history of PVLs may mimic that of native valve regurgitation. Uncorrected hemolysis eventually results in severe anemia.

\section{Clinical Findings}

Patients with symptomatic PVLs present with congestive heart failure in over $90 \%$ of cases. Most report NYHA Class III or greater symptoms $[19,20]$. Clinical presentation may occur immediately after surgery or significantly later [21]. Hemolytic anemia is present between $30-75 \%$ of cases referred for intervention $[19,20]$.

The regurgitation associated with large PVLs is often associated with a murmur on cardiac auscultation. In para-mitral valve leaks, a blowing, holosystolic murmur is typically heard radiating to the axillae. However, paravalvular regurgitatant jets may be oriented differently than jets associated with intra-valvular regurgitation; if the jet is oriented posteriorly, radiation may be noted in the back. If the jet is oriented anteriorly, radiation to the base may be heard. The murmur appreciated in para-aortic valve leaks is typically a blowing, decrescendo, diastolic murmur that is heard best at the left sternal border with the patient sitting forward and in end-expiration.

The majority of patients presenting with symptomatic PVLs have elevations of $\mathrm{N}$-terminal pro-brain natriuretic peptide. Brain natriuretic peptide is typically elevated in patients with congestive heart failure, but it has also been shown to correlate with the severity and symptoms of aortic and mitral regurgitation $[22,23]$. When hemolytic anemia is present, laboratory studies will show decreased hemoglobin, markedly elevated lactate dehydrogenase, markedly decreased haptoglobin, and increased indirect bilirubin.

\section{Echocardiography}

Transesophageal (TEE) and transthoracic (TTE) echocardiography should be used to assess pros-

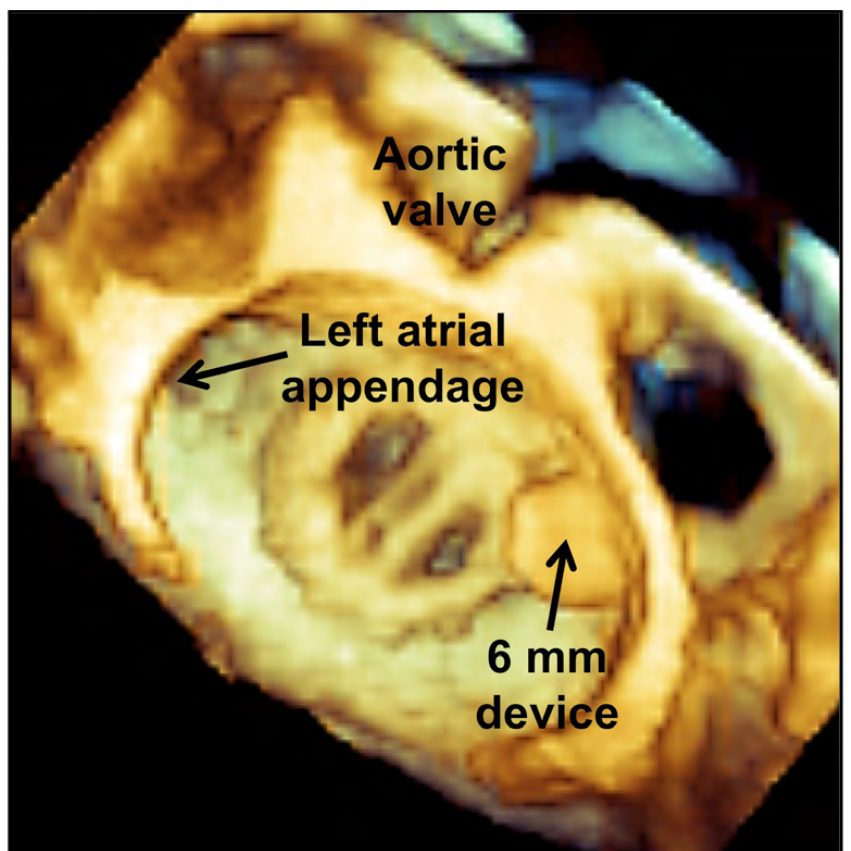

Figure 4. $3 \mathrm{D}$ full-volume rendering of the mechanical mitral prosthesis with 6-mm occluder device in position. Aortic valve is in the 12 o'clock position.

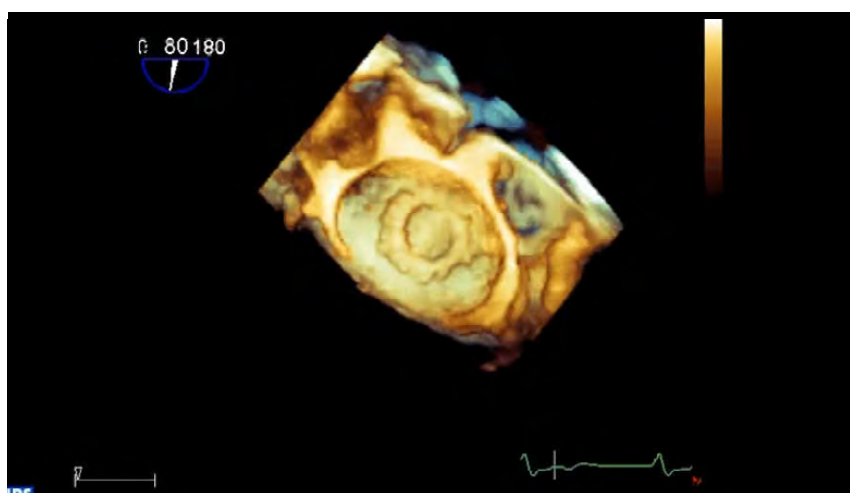

Video 4. Case 1: 3D acquisition of $6 \mathrm{~mm}$ occluder device expansion.

thetic valve function and the spatial characteristics of PVLs. Color Doppler can help identify the location, direction, and severity of regurgitant blood flow. However, because the spatial resolution of traditional TEE and TTE is limited, the addition of 3D allows for improved spatial resolution and therefore provides more information regarding the size and shape of PVLs $[24,25]$. This information is especially helpful during percutaneous closure procedures. RT3D TEE allows for operators to visualize the length of the 


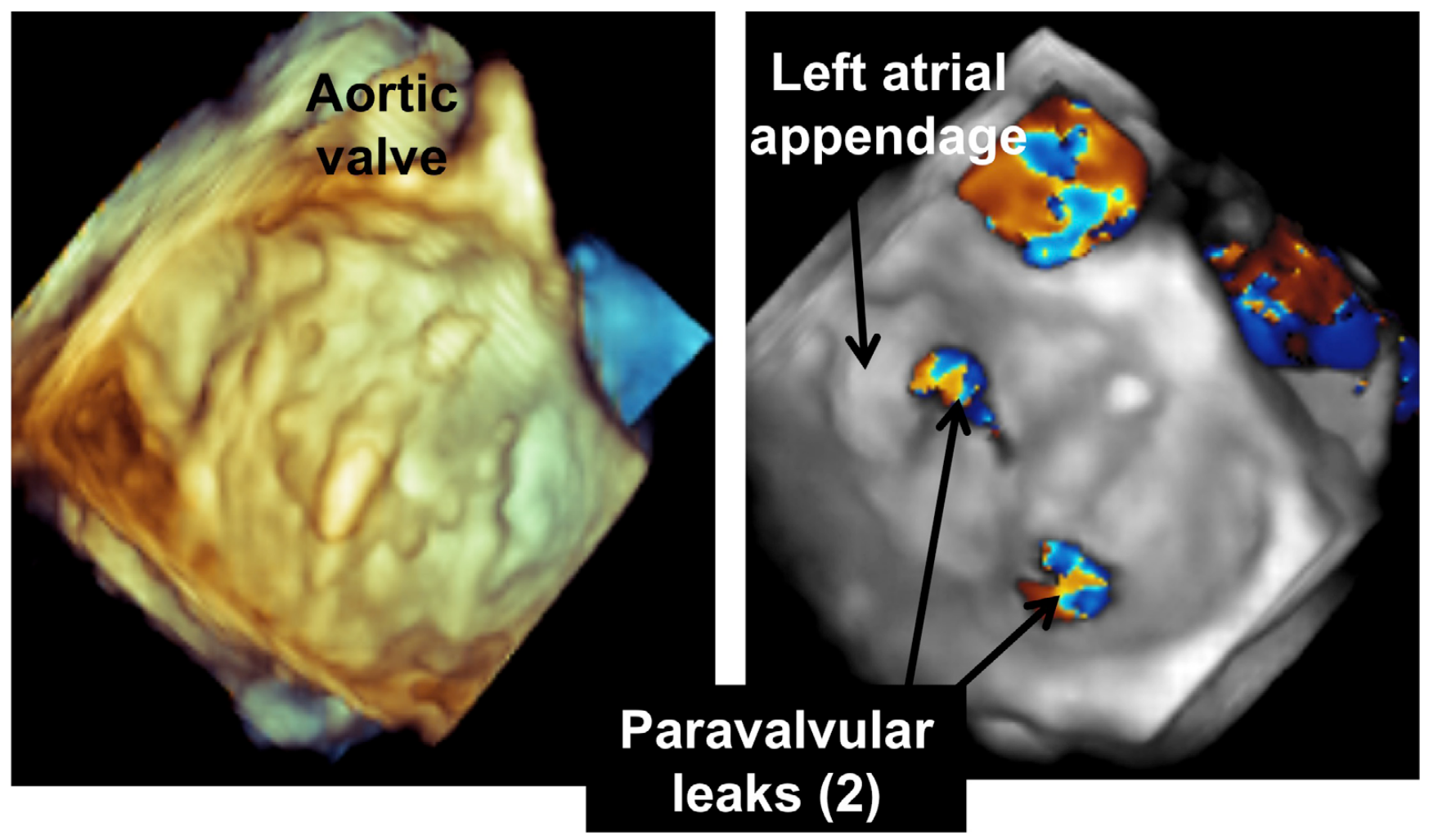

Figure 5. 3D zoomed rendering of the mechanical mitral prosthesis (left panel). With this modality, it is difficult to appreciate the two disks associated with the valve prosthesis in this patient. The paravalvular leaks are not seen. The 3D color zoom acquisition of the mitral prosthesis allows better appreciation of the location and number of paravalvular leaks (right panel). This patient has two paravalvular leaks. Aortic valve is in the 12 o'clock position.

catheter or guidewire, identify the size, shape, and number of PVL, and ensure that any deployed closure device does not impair movement of the mechanical valve leaflets.

The majority of PVL are crescentic, oval, or round in shape. Their track can be parallel, perpendicular, or serpiginous in relation to the direction of prosthetic blood flow. The most common location for mitral PVLs are along the posterior wall (5-6 o'clock from the surgeon's perspective) and along the aortic-mitral curtain (10-11 o'clock) [19,26]. The prevalence of PVLs in the posterior mitral annulus has been attributed to the following: (1) the posterior annulus provides a limited surgical field view for suturing (2) the proximity of the circumflex artery may lead to more superficial suturing, and (3) calcification and fibrosis are more prevalent in the posterior annulus [24].

The recommended methods used to assess the severity of para-mitral valve regurgitation are similar to those used to evaluate native mitral regurgitation. Color flow regurgitant jet area, jet density, and systolic pulmonary venous flow reversal are all recommended in the assessment of para-mitral valve regurgitation [27]. The proportion of the circumference of the sewing ring occupied by the regurgitatant jet provides an approximate guide to severity, with more than $20 \%$ indicating severe regurgitation and less than $10 \%$ consistent with mild regurgitation [27]. The proximal isovelocity surface area (PISA) measurement has not been validated in paravalvular regurgitation, but large PISA shell measurements of paravalvular regurgitant jets have been reported to be more consistent with severe regurgitation [28]. Jet eccentricity may limit traditional assessment with color Doppler.

Aortic PVLs are more commonly located in the vicinity of the non-coronary or right coronary cusps [29]. Para-aortic regurgitation is also assessed with accepted criteria that are used to assess native aor- 
tic insufficiency. Typical criteria include pressure halftime, jet width, jet density, and diastolic flow reversal in the descending aorta [27].

There are several challenges associated with assessment of paravalvular regurgitation with echocardiography. Mechanical valves create significant image distortion due to acoustic shadowing, and showing may actually hide the presence of regurgitant jets. When multiple PVLs are present, echocardiographic assessment is difficult due to eccentric regurgitant jets and absence of validated echocardiographic parameters.

\section{Other Imaging Techniques}

PVLs may also be evaluated with EKG-gated computed tomographic angiography (CTA). These images can be retrospectively reconstructed to form 4D-reconstructions, that allow for detailed visualization of PVLs. These images have been used to assist planning for percutaneous PVL closure procedures [19]. Like echocardiography, CTA is limited by artifact from high-density structures like the prosthetic valve and extensive calcification. In addition, CTA requires IV contrast and radiation exposure, therefore, the risk of IV contrast and radiation exposure must therefore be weighed against the potential benefit.

Angiography has historically been used to assess the location, size, and hemodynamic severity of PVLs. However, it is difficult to determine the 3D anatomic and spatial characteristics of the defect with angiography, alone. Invasive assessment of the PVL with test balloons to assess PVL size, distensibility, and hemodynamic implications of closure is no longer recommended due to the risk of balloon entrapment.

\section{Treatment}

Medical therapy in large PVLs is directed at symptom reduction by either treating the heart failure or treating the anemia caused by hemolysis. Despite these interventions, the majority of patients with severe $P V L$ require definitive, structural correction via either open surgery or transcatheter-based intervention.

Until recently, surgical management of PVLs was the only available treatment for severe disease. Surgical correction improves overall survival and symptoms in patients with severe PVL, when compared

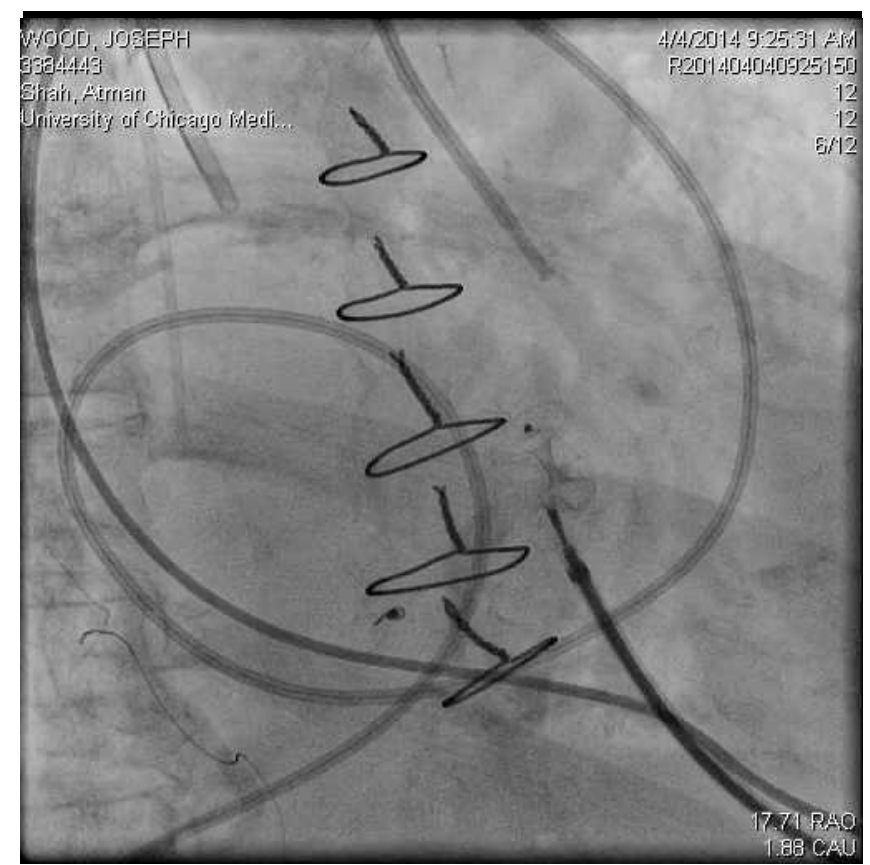

Video 5. Case 2: Fluoroscopy showing evidence of entrapment of the posterior mechanical mitral valve leaflet.

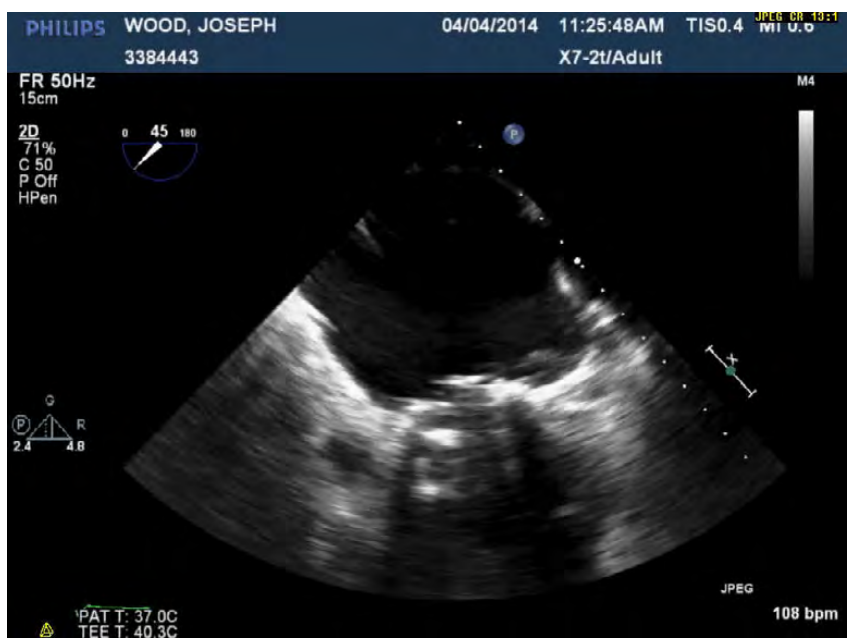

Video 6. Case 2: 2DTEE showing evidence of entrapment of the posterior mechanical mitral valve leaflet.

to medical therapy, alone [12]. Surgery entails either repair of the PVL or re-do replacement of the prosthetic valve. Many approaches to surgical correction of mitral PVLs have been described, but most involve either direct suturing, patching, or incorporation of autologous tissue from neighboring structures [3034]. The choice of repair versus replacement depends 


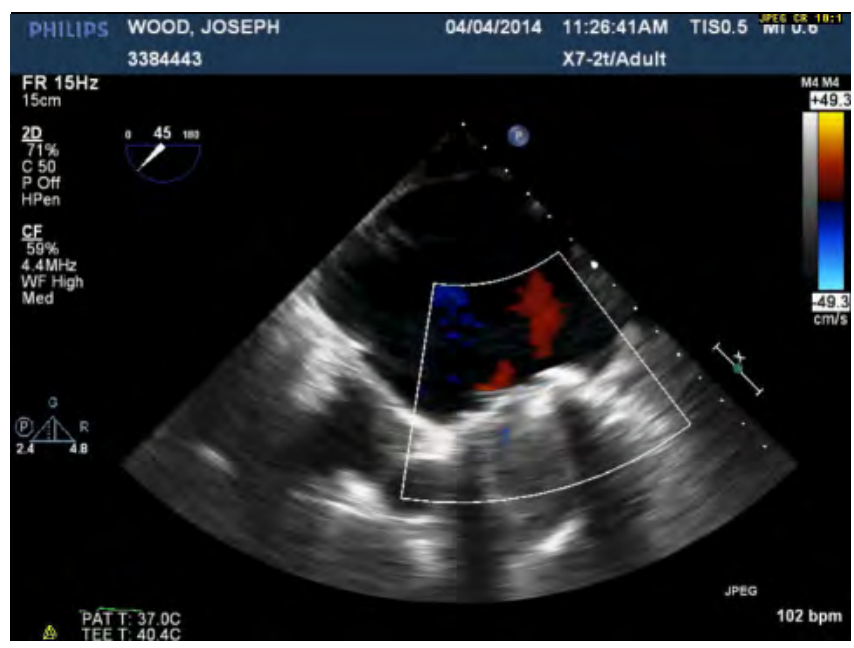

Video 7. Case 2: 2D color Doppler TEE showing evidence of entrapment of the posterior mechanical mitral valve leaflet.

largely on the specific etiology of PVL, location, and size of the leak. Operative mortality for surgery to replace a dysfunctional mechanical or bioprosthetic valve is $5 \%$ to $14 \%[35,36]$. Hospital mortality has been described as $13 \%$ for initial re-operation, with subsequent operations associated with significantly higher mortality [37].

Since first described in 1992, percutaneous transcatheter closure of PVLs has become an attractive alternative to surgical correction [3]. Advancement of real-time 3DTEE imaging has contributed to the success of catheter-based techniques. These procedures do not require cardio-pulmonary bypass, and therefore may carry a lower risk than traditional surgery. A variety of techniques have been described in the literature [1,3-9].

Percutaneous PVL repair may be performed from multiple access points; retrograde via the femoral or radial artery, antegrade via femoral vein (with trans-septal perforation to access the left heart), or directly via trans-apical puncture [38]. The specific access site and approach is determined in a case-bycase basis with consideration for the location of the defect, location of the prosthesis, other anatomical considerations, multiple patient-specific issues, and operator experience.

Closure of aortic PVL is typically performed via retrograde arterial approach. A guidewire is advanced through the leak, with real-time 3DTEE and fluoroscopy used to ensure that the wire is crossing the PVL.
The size and shape of the defect, typically evaluated by echocardiography determines the size of the delivery catheter used. The occlusion device is then loaded onto the delivery catheter, advanced into position, and deployed. Before and after release of the occlusion device, the operator must confirm free motion of the prosthetic leaflets, stable anchoring of occlusion device, and reduction of the regurgitant jet [38].

Mitral PVL closure is technically more challenging than aortic PVL closure. It is typically performed using the femoral venous trans-septal approach. Transseptal puncture typically requires simultaneous TEE or intracardiac ultrasound to minimize the risk of complication. The location of the PVL itself along the mitral annulus determines the optimal approach for the procedure. For example, if the PVL is close to the atrial septum, it may be difficult to engage the PVL via the femoral venous trans-septal approach. Furthermore, retrograde arterial approach may be needed to snare the wire placed via the trans-septal approach to provide a more stable rail for device deployment. However, left ventricular structures (such as trabeculae, papillary muscles, and chordae) may complicate retrograde engagement of mitral PVRs. In some instances, access via a trans-apical approach is required. This approach provides direct engagement of mitral PVL at any location around the mitral annulus. It is typically achieved with surgical access and direct visualization of the left ventricular apex, although fully percutaneous trans-apical access is possible, as shown in the above cases $[39,40]$.

At this time, the majority of percutaneous PVR repairs are performed with Amplatzer devices (St. Jude Medical, St. Paul, Minnesota, USA), although vascular coils have also been used [1,20,25,41-43]. The devices used are either cylindrical or oval in shape. The success of percutaneous PVL repair hinges on proper selection of occlusion devices. Selection is predicated on the size and shape of the PVL. Because most PVL are oval in shape, oval occlusion devices may be preferred in most cases. Large PVL require large occlusion devices. Unfortunately, larger occlusion devices increase the risk for prosthetic leaflet impingement, because the discs of the device can overhang the sewing ring. Some authors have suggested that this risk may be alleviated by placing multiple smaller occlusive devices in the large defect [38]. 

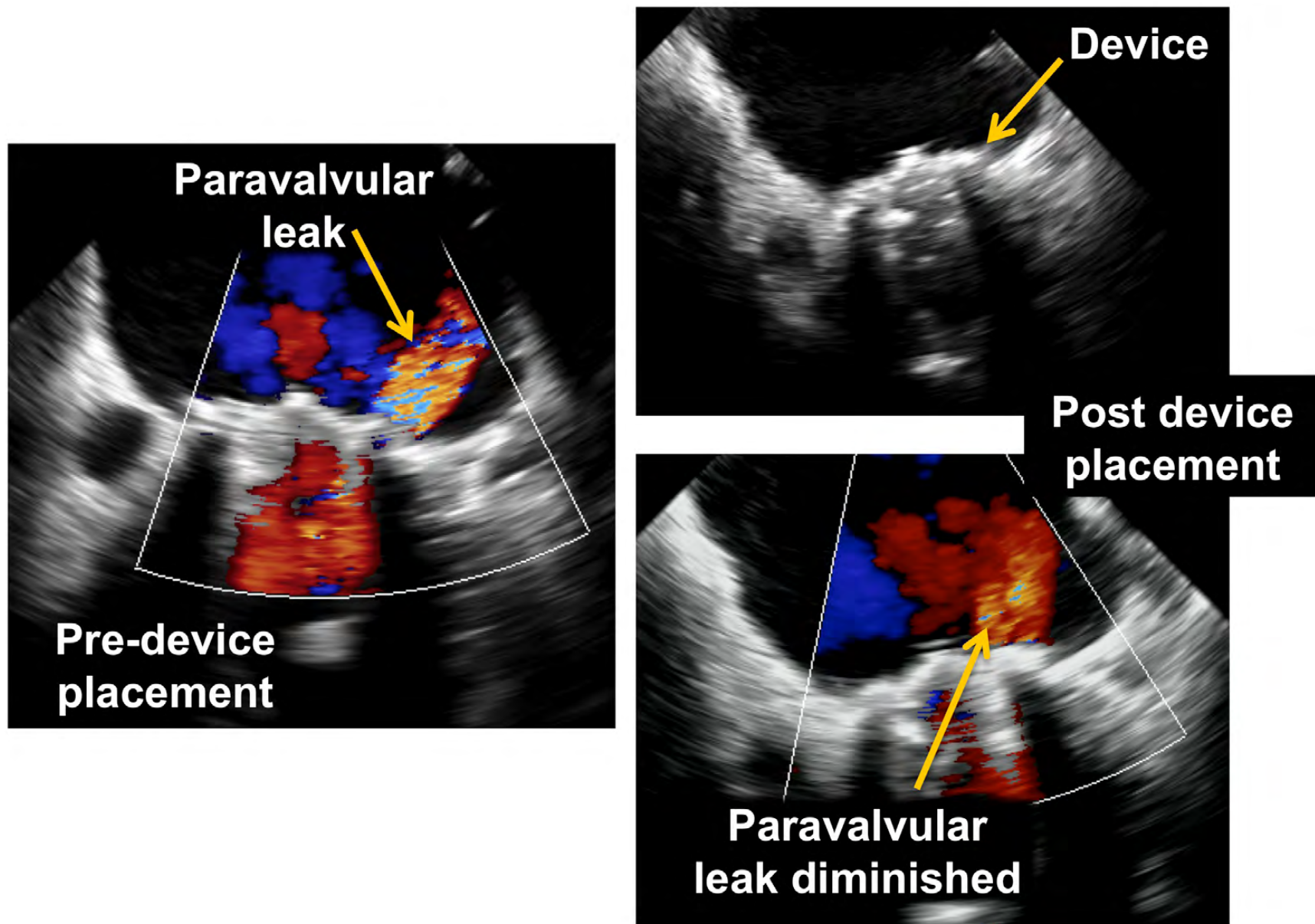

Figure 6. 2D TEE images of the paravalvular leak that was closed with device (left panel). The top right image shows the device in situ and the bottom right image shows diminished significance of this paravalvular leak post device placement.

Percutaneous PVL closure has a technical success rates of 77 to $88 \%$ in high-volume centers, with some reporting success rates greater than $95 \%$ $[20,25,40,42]$. Clinically, significant success has been reported from 67 to $77 \%$ of cases. Peri-procedure complication rates have been reported around $10 \%$, with a mortality of approximately $1 \%$. Peri-procedural complications include cardiac tamponade, device embolization, damage to prosthetic valve, and stroke. Late embolization of occlusive devices has been reported, but is rare $[44,45]$.

\section{Conclusions}

Symptomatic PVL is an uncommon, but serious complication of surgical valve replacement. Assess- ment of the severity of PVL requires thoughtful interpretation of clinical presentation and multiple imaging modalities. Once identified, successful closure of symptomatic PVL can be achieved with surgical re-operation or percutaneous closure from a variety of approaches. As highlighted in the two cases, real-time 3D TEE is invaluable in guiding the successful percutaneous closure of PVL.

\section{Conflict of Interest}

The authors have no conflict of interest relevant to this publication.

Comment on this Article or Ask a Question 


\section{References}

1. Pate GE, Al Zubaidi A, Chandavimol M, Thompson CR, Munt Bl, Webb JG. Percutaneous closure of prosthetic paravalvular leaks: Case series and review. Catheter Cardiovasc Interv. 2006;68:528-533. DOI: $10.1002 / \mathrm{ccd} .20795$

2. Miller DL, Morris JJ, Schaff H V, Mullany CJ, Nishimura RA, OrszulakTA. Reoperation for aortic valve periprosthetic leakage: identification of patients at risk and results of operation. J. Heart Valve Dis. 1995;4:160-165. PMID: 8556176

3. Hourihan M, Perry SB, Mandell VS, Keane JF, Rome JJ, Bittl JA, et al. Transcatheter umbrella closure of valvular and parazvalvular leaks. J Am Coll Cardiol. 20, 1371-1377 (1992). DOI: 10.1016/0735-1097(92)90250-Q

4. Kort HW, Sharkey AM, Balzer DT. Novel use of the Amplatzer duct occluder to close perivalvar leak involving a prosthetic mitral valve. Catheter. Cardiovasc. Interv. 2004;61(4):548-551. DOI: 10.1002/ ccd. 10785

5. Moscucci M, Deeb GM, Bach D, Eagle KA, Williams DM. Coil embolization of a periprosthetic mitral valve leak associated with severe hemolytic anemia. Circulation. 2001;104(16):e85-e86. DOI: 10.1161/ hc4101.096829

6. Webb JG, Pate GE, Munt BI. Percutaneous closure of an aortic prosthetic paravalvular leak with an Amplatzer duct occluder. Catheter Cardiovasc Interv. 2005;65(1):6972. DOI: $10.1002 / \mathrm{ccd} .20337$

7. Hildick-Smith D, Behan MWH, De Giovanni J. Percutaneous closure of an aortic paravalvular leak via the transradial approach. Catheter Cardiovasc Interv. 2007;69:708710. DOI: $10.1002 / \mathrm{ccd} .21043$

8. Rihal CS, Sorajja P, Booker JD, Hagler DJ, Cabalka AK. Principles of percutaneous paravalvular leak closure. JACC Cardiovasc Interv. 2012;5:121-130. DOI: 10.1016/j. jcin.2011.11.007

9. Cortés M, García E, García-Fernandez MA, Gomez JJ, Perez-David E, Fernández-Avilés F. Usefulness of transesophageal echocardiography in percutaneous transcatheter repairs of paravalvular mitral regurgitation. Am J Cardiol. 2008;101:382-386. DOI: 10.1016/j.amjcard.2007.08.052

10. Hammermeister K, Sethi GK, Henderson WG, Grover FL, Oprian C, Rahimtoola SH. Outcomes 15 years after valve replacement with a mechanical versus a bioprosthetic valve: Final report of the Veterans Affairs randomized trial. J Am Coll Cardiol. 2000;36(4):1152-1158. DOI: 10.1016/ S0735-1097(00)00834-2
11. Ionescu a, Fraser a G, Butchart EG. Prevalence and clinical significance of incidental paraprosthetic valvar regurgitation: a prospective study using transoesophageal echocardiography. Heart. 2003;89(11):1316-1321. DOI: 10.1136/ heart.89.11.1316

12. Genonia $M$, Franzena $D$, Vogta $P$, Seifertb $B$, Jennic R, Künzlia A, et al. Paravalvular leakage after mitral valve replacement: improved long-term survival with aggressive surgery? Eur J Cardiothorac Surg. 2000;17(1):14-19. DOI: 10.1016/S10107940(99)00358-9

13. Safi AM, Kwan T, Afflu E, Al Kamme A, Salciccioli L. Paravalvular regurgitation: a rare complication following valve replacement surgery. Angiology. 2000;51:479-487. DOI: 10.1177/000331970005100605

14. Genoni M, Franzen D, Tavakoli R, Seiffert B, Graves K, Jenni R, et al. Does the morphology of mitral paravalvular leaks influence symptoms and hemolysis? J Heart Valve Dis. 2001;10:426-430. PMID: 11499584

15. Wąsowicz M, Meineri M, Djaiani G, Mitsakakis N, Hegazi N, Xu W, et al. Early complications and immediate postoperative outcomes of paravalvular leaks after valve replacement surgery. J Cardiothorac Vasc Anesth. 2011;25:610-614. DOI: 10.1053/j. jvca.2011.01.012

16. Kliger C, Eiros R, Isasti G, Einhorn B, Jelnin V, Cohen $\mathrm{H}$, et al. Review of surgical prosthetic paravalvular leaks: Diagnosis and catheter-based closure. Eur Heart J. 2013;34:638648. DOI: 10.1093/eurheartj/ehs347

17. Melacini P, Villanova $C$, Thiene G, Minarini M, Fasoli G, Bortolotti U, et al. Long-term echocardiographic Doppler monitoring of Hancock bioprostheses in the mitral valve position. Am J Cardiol. 1992;70:1157-1163. DOI: 10.1016/0002-9149(92)90048-4

18. Duvernoy WF, Gonzalez-Lavin L, Anbe DT. Spontaneous closure of paravalvular leak after mitral valve replacement. Chest. 1975;68:102-104. DOI: 10.1378/ chest.68.1.102

19. Ruiz CE, Jelnin V, Kronzon I, Dudiy Y, Del Valle-Fernandez R, et al. Clinical outcomes in patients undergoing percutaneous closure of periprosthetic paravalvular leaks. J Am Coll Cardiol. 2011;58(21):2210-2217. DOI: 10.1016/j.jacc.2011.03.074

20. Sorajja P, Cabalka AK, Hagler DJ, Rihal CS. Percutaneous repair of paravalvular prosthetic regurgitation: Acute and 30-day outcomes in 115 patients. Circ Cardiovasc Interv. 2011;4(4):314-321. DOI: 10.1161/ CIRCINTERVENTIONS.110.960955
21. Misawa Y, Saito T, Konishi H, Oki S, Kaminishi $Y$, Takahashi $H$, et al. When and how does nonstructural mechanical prosthetic heart valve dysfunction occur? Jpn J Thorac Cardiovasc Surg. 2003;51:355-360. DOI: 10.1007/BF02719467

22. Gerber IL, Stewart RAH, French JK, Legget ME, Greaves SC, West TM, et al. Associations between plasma natriuretic peptide levels, symptoms, and left ventricular function in patients with chronic aortic regurgitation. Am J Cardiol. 2003;92:755-758. DOI: 10.1016/S0002-9149(03)00849-X

23. Sutton TM, Stewart RAH, Gerber IL, West TM, Richards AM, Yandle TG, et al. Plasma natriuretic peptide levels increase with symptoms and severity of mitral regurgitation. J Am Coll Cardiol. 2003;41:2280-2287. DOI: 10.1016/S0735-1097(03)00486-8

24. Kronzon I, Sugeng L, Perk G, Hirsh D, Weinert L, Fernandez MAG, et al. Real-time 3-dimensional transesophageal echocardiography in the evaluation of post-operative mitral annuloplasty ring and prosthetic valve dehiscence. J Am Coll Cardiol. 2009;53(17):1543-1547. DOI: 10.1016/j.jacc.2008.12.059

25. García-Fernández MA, Cortés M, García-Robles JA, Gomez de Diego JJ, Perez-David E, García E. Utility of real-time three-dimensional transesophageal echocardiography in evaluating the success of percutaneous transcatheter closure of mitral paravalvular leaks. J Am Soc Echocardiogr. 2010;23(1):26-32. DOI: 10.1016/j. echo.2009.09.028

26. De Cicco G, Russo C, Moreo A, Beghic C, Fuccia C, Gerometta $P$, et al. Mitral valve periprosthetic leakage: anatomical observations in 135 patients from a multicentre study. Eur J Cardiothora Surg. 2006;30(6):887-891. DOI: 10.1016/j. ejcts.2006.09.019

27. Zoghbi W a, Chambers JB, Dumesnil JG, Foster E, Gottdiener JS, Grayburn PA, et al. Recommendations for evaluation of prosthetic valves with echocardiography and doppler ultrasound: a report From the American Society of Echocardiography's Guidelines and Standards Committee and the Task Force on Prosthetic Valves, developed in conjunction. J Am Soc Echocardiogr. 2009;22(9):975-1014; quiz 1082-4. DOI: 10.1016/j.echo.2009.07.013

28. Kliger $C$, Eiros $R$, Isasti $G$, Einhorn $B$, Jelnin $V$, Cohen $H$, et al. Review of surgical prosthetic paravalvular leaks: diagnosis and catheter-based closure. Eur Heart J. 2013;34(9):638-649. DOI: 10.1093/eu- 
rheartj/ehs347

29. De Cicco G, Lorusso R, Colli A, Nicolini F, Fragnito $C$, Grimaldi T, et al. Aortic valve periprosthetic leakage: Anatomic observations and surgical results. Ann Thorac Surg. 2005;79(5):1480-1485. DOI: 10.1016/j. athoracsur.2004.11.058

30. Moneta A. An alternative technique for non-infective paraprosthetic leakage repair. Eur J CardioThora Surg. 2003;23(6):1074-1075. DOI: 10.1016/ S1010-7940(03)00163-5

31. Al Halees Z. An additional maneuver to repair mitral paravalvular leak. Eur J Cardiothorac Surg. 2011;39(3):410-1. DOI: 10.1016/j.ejcts.2010.06.028

32. Lafci G, Gedik HS, Korkmaz K, Yalcinkaya A, Yasar E, Cagli K. Repair of mitral paravalvular leak with double left atrial wall folding. Gen Thorac Cardiovasc Surg. 2013;61:483484. DOI: $10.1007 / \mathrm{s} 11748-013-0228-2$

33. Ali I. Intraoperative precise localization of paravalvular mitral leak by a simple surgical maneuver. J Card Surg. 2008;23:541-542. DOI: 10.1111/j.1540-8191.2008.00609.x

34. Ozkan M, Aykan AC, Gursoy O, Tuncer MA, Cakal B, Yildiz M, et al. An alternative approach for mitral paravalvular leak with dacron patch curtaining under the guidance of real-time 3-dimensional transesophageal echocardiography. Cardiology. 2011;119:34-37. DOI: 10.1159/000329843

35. Toker ME, Kirali K, Balkanay M, Eren E, Özen $Y$, Güler $M$, et al. Operative mortality after valvular reoperations. Heart Surg Forum. 2005;8:E280-E283; discussion E283. DOI: 10.1532/HSF98.20041154
36. Potter DD, Sundt TM, Zehr KJ, Dearani JA, Daly RC, Mullany $C J$, et al. Risk of repeat mitral valve replacement for failed mitral valve prostheses. Ann Thorac Surg. 2004;78(1):67-72; discussion 67-72. DOI: 10.1016/j.athoracsur.2004.02.014

37. Echevarria JR, Bernal JM, Rabasa JM, Morales $D$, Revilla Y, Revuelta JM. Reoperation for bioprosthetic valve dysfunction. A decade of clinical experience. Eur J Cardiothorac Surg. 1991;5:523-526; discussion 527. DOI: $10.1016 / 1010-7940(91) 90105-S$

38. Binder RK, Webb JG. Percutaneous mitral and aortic paravalvular leak repair: indications, current application, and future directions. Curr Cardiol Rep. 2013;15:342. DOI: 10.1007/s11886-012-0342-2

39. Martinez CA, Rosen R, Cohen H, Ruiz CE. A novel method for closing the percutaneous transapical access tract using coils and gelatin matrix. J Inv Cardiol. 2010;22:E107-E109. PMID: 20516515

40. Nietlispach F, Eckstein F, Seeberger M, Osswald S, Kaufmann BA, Reuthebuch O. Closure of apical access site after transapical, transcatheter paravalvular leak closure. Can J Cardiol. 2012;28. DOI: 10.1016/j. cjca.2012.01.023

41. Shapira $Y$, Hirsch R, Kornowski R, Hasdai $D$, Assali A, Vaturi $M$, et al. Percutaneous closure of perivalvular leaks with Amplatzer occluders: feasibility, safety, and shortterm results. J Heart Valve Dis. 2007;16:305-313. PMID: 17578053

42. Ruiz CE, Jelnin V, Kronzon I, Dudiy Y, Del Valle-Fernandez R, Einhorn BN, et al. Clinical outcomes in patients undergoing percuta- neous closure of periprosthetic paravalvular leaks. J Am Coll Cardiol. 2011;58:22102217. DOI: 10.1016/j.jacc.2011.03.074

43. Moore DJ, Lashus AG, Prieto LR, Drummond-Webb J, Latson LA. Transcatheter coil occlusion of perivalvular mitral leaks associated with severe hemolysis. Catheter Cardiovasc Interv.2000;49:64-67.DOI:10.1002/ (SICI) 1522-726X(200001)49:1<64::AIDCCD14>3.0.CO;2-C

44. Arzamendi D, Li CH, Serra A. Late embolization of a vascular plug III device after mitral paravalvular leak closure. Catheter Cardiovasc Interv. 2013;82. DOI: 10.1002/ ccd.25039

45. Kennedy JLW, Mery CM, Kern JA, Bergin JD. Mitral stenosis caused by an amplatzer occluder device used to treat a paravalvular leak. Ann Thorac Surg. 2012;93:2058-2060. DOI: 10.1016/j.athoracsur.2011.11.064

Cite this article as: Venturini JM, Mediratta A, Addetia K, Nathan S, Shah AP, Lang RM. Real-Time 3D Transesophageal Echocardiographic Guidance of Prosthetic Valve Paravalvular Leak. Structural Heart Disease 2015;1(1): 9-19. DOI: http://dx.doi.org/10.12945/j. jshd-.2015.00011-14 